\title{
High-Density Data Storage Based on the Atomic Force Microscope
}

\author{
H. JONATHON MAMIN, ROBERT P. RIED, MEMBER, IEEE, \\ BRUCE D. TERRIS, AND DANIEL RUGAR, MEMBER, IEEE
}

\section{Invited Paper}

The atomic force microscope (AFM), with its ability to image and modify surfaces on the nanometer scale, offers the potential for simple, compact, high-density data-storage devices. At the heart of the technique is a microfabricated cantilever with a sharp tip on the end. Using modern micromachining techniques, it is possible to batch fabricate cantilevers with tips that are sharp on the scale of $100 \AA$. We have pursued a particular AFM storage scheme based on mechanical readback of topographic data using highfrequency, piezoresistive silicon cantilevers. Areal densities of 65 Gbit/in ${ }^{2}$ have been demonstrated, with readback rates greater than $10 \mathrm{Mbit} / \mathrm{s}$. Nanoreplication techniques have been used to produce read-only disks. In addition, a write-once scheme was developed that uses integrated heating elements on the cantilevers in order to perform thermomechanical writing on a polymer substrate. Considerable progress has been made in addressing critical issues such as data rate, reliability, and practical implementation, but significant challenges still remain, both in the technology and in finding the most suitable applications.

Keywords-Atomic force microscope, memories, microelectromechanical devices, piezoresistive devices.

\section{INTRODUCTION}

The history of the information age has been characterized by rapid advances in the underlying component technologies, leading to continuous improvements in computing power at ever-decreasing cost. Much of the benefit has arisen from the power of scaling, as exemplified by the well-known Moore's Law. In mass data storage based on magnetic recording, the progress has been just as dramatic as in semiconductor devices. In the past 40 years, the areal density of disk drives has increased by six orders of magnitude [1]. Recent laboratory demonstrations made at an areal density of $12 \mathrm{Gbit} / \mathrm{in}^{2}$ suggest that progress will continue for some time [2]. History is, in fact, littered

Manuscript received November 19, 1998; revised February 16, 1999. This work was supported in part by DARPA Contract DABT-63-95-C0019.

H. J. Mamin, B. D. Terris, and D. Rugar are with the IBM Research Division, Almaden Research Center, San Jose, CA 95120-6099 USA.

R. P. Ried is with Input/Output, Inc., Stafford, TX 77477-2409 USA. Publisher Item Identifier S 0018-9219(99)04046-3. with failed predictions of the demise of magnetic recording. Still, one can be fairly certain that densities for magnetic recording will not increase another six orders of magnitude, as this would require bit cells of atomic dimensions. Indeed, at densities in the range of $100 \mathrm{Gbit} / \mathrm{in}^{2}$ (which are less than ten years away at the current rate of progress [1]), superparamagnetic effects, in which the magnetic bits are no longer stable at room temperature, are expected to pose serious challenges. It is therefore prudent to make some examination of technologies that could have the potential to provide a pathway to the higher densities needed in the years ahead.

Ever since the invention of the scanning tunneling microscope (STM) [3], with its ability to image surfaces at the atomic scale, people have envisioned the possibility of applying the technique to perform ultrahigh-density data storage [4]. A wide variety of schemes have been demonstrated for using the STM and its offspring, the atomic force microscope (AFM) [5], to modify as well as image surfaces with spatial resolution ranging from angstroms to tens of nanometers [6], [7]. Much of the work has focused on fundamental understanding of the mechanisms and interactions between the tip and sample. In the case of AFM-based storage, work toward a tipbased data-storage system has progressed beyond simply demonstrating the ability to make a small mark. Research is underway to address such critical issues as data rate, error rates, tracking, integration, and reliability. It is this work that is the main subject of this paper.

\section{GENERAL CONSIDERATIONS FOR SCANNING PROBE DATA StORAGE}

\section{A. STM-Based Data Storage}

In the STM, a sharp tip is scanned in close proximity to a surface. An applied voltage between the tip and sample gives rise to a tunnel current that depends sensitively on the tip-sample separation. As the tip is scanned, the 


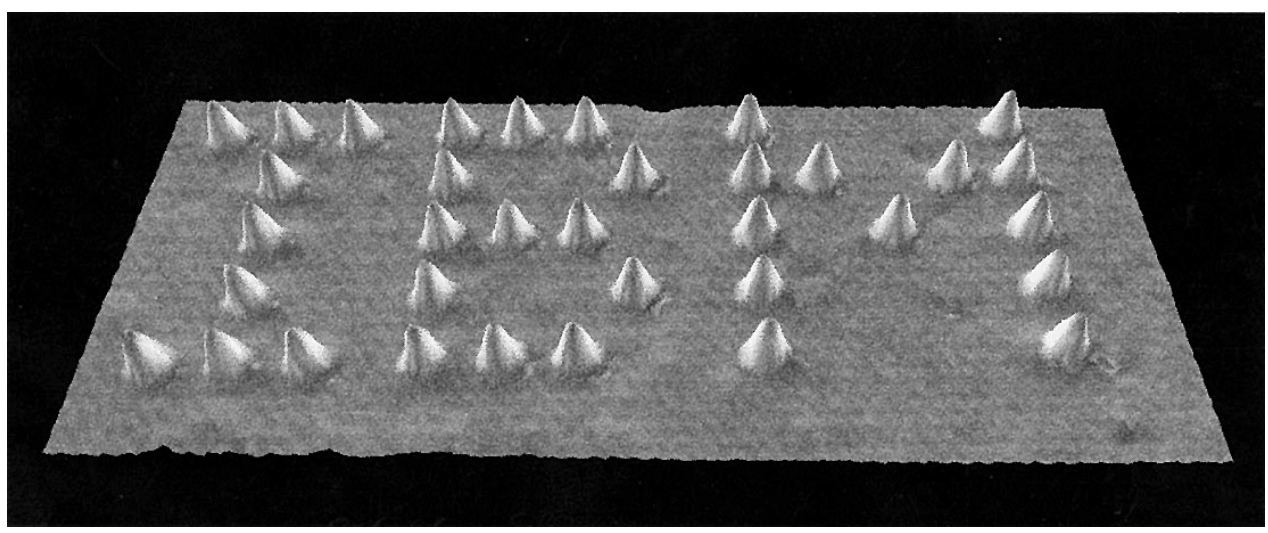

Fig. 1. Atomic-scale message demonstrating the ability to position single atoms with angstom precision. Each bump is an individual xenon atom positioned with the tip of a scanning tunneling microscope operating at $4.2 \mathrm{~K}$. This represents perhaps the ultimate in data-storage density, with a single atomic "bit" (courtesy of D. Eigler, IBM Research Division, Almaden Research Center).

tunnel current is monitored and the tip position is servoed to maintain constant current. In this way, the underlying surface topography is mapped out [3]. From a data-storage perspective, the chief advantage of this approach, and perhaps the only reason it is worth contemplating, is that one can truly image and modify surfaces with the tip on a nanometer, or even atomic, scale. A famous example of this is shown in Fig. 1, where individual xenon atoms were positioned with angstrom precision using an STM to spell out "IBM." This seminal work by Eigler and Schweizer was performed in ultrahigh vacuum (UHV) at $4.2 \mathrm{~K}$, and took

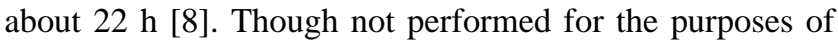
data storage, this demonstration represents an effective areal density of the order of $10^{15} \mathrm{bits} / \mathrm{in}^{2}$, or $1 \mathrm{million} \mathrm{Gbit} / \mathrm{in}^{2}$ $\left(1.6 \times 10^{14} \mathrm{bit} / \mathrm{cm}^{2}\right)$.

The STM has some characteristics, however, that would tend to seriously limit the speed and reliability of an STMbased storage device. Because of the steep dependence of the tunnel current on separation, the tip-sample separation must be maintained to within about $0.1 \AA$ to achieve reasonable current stability. Using an active servo to do this with any reasonable speed is difficult. There has been some progress in improving the bandwidth of the STM using novel mechanics and servo schemes [9], but such improvements have not come easily. The possibility of operating without a servo has often been proposed, but suitable samples that have the required degree of flatness over large scales have yet to be demonstrated.

In addition, the STM suffers from the difficulty of its extreme dependence on the surface properties (e.g., workfunction, oxidation state, cleanliness, etc.), and the exact configuration of the tip. These effects give rise to large and unpredictable changes in tunnel current, especially when operating under less than UHV conditions. For these reasons, much of the work in data-storage applications has focused on the atomic force microscope implementation.

\section{B. AFM-Based Data Storage}

In the AFM, the sharp tip sits on the end of a soft cantilever spring, so that extremely small forces are capable

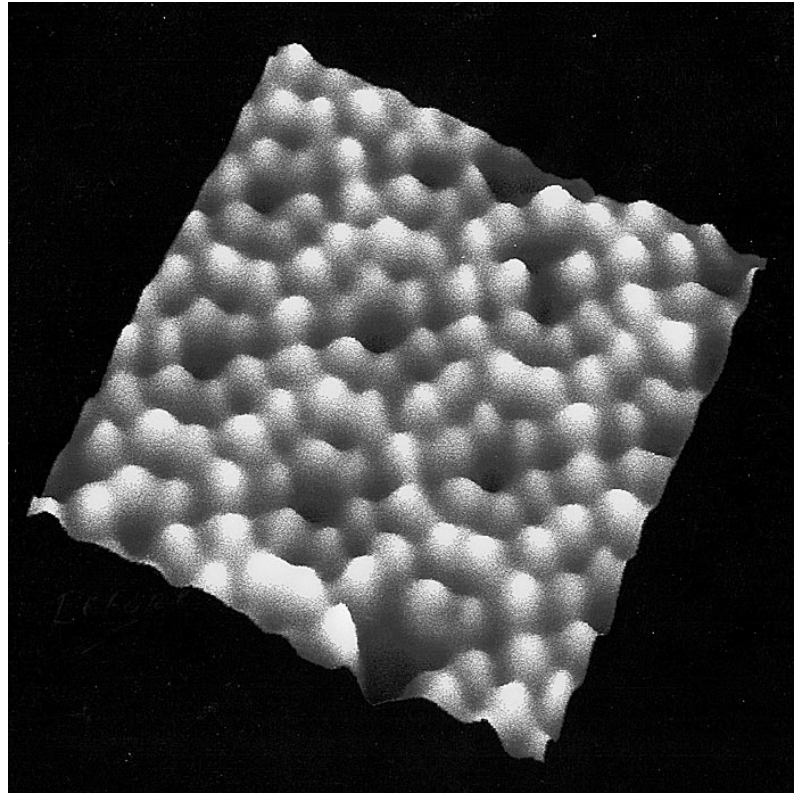

Fig. 2. Image obtained with the AFM showing individual silicon adatoms on a clean reconstructed Si (111) surface (courtesy of R. Erlandsson, Linkoping University, Sweden).

of being sensed and applied [5]. The advantage is that the tip can actually "touch" the surface, i.e., come into repulsive contact with it, without necessarily destroying the tip or surface, due to the compliance of the cantilever. The tip can also operate in a noncontact mode, in which it responds to the attractive or repulsive forces exerted on it by the surface.

As with the STM, the AFM is capable of true atomic resolution. An example is shown in Fig. 2, in which individual silicon adatoms in the famous $\mathrm{Si}(7 \times 7)$ surface reconstruction have been imaged on a clean surface in UHV using noncontact mode AFM [10]. This remarkable ability to access the atomic scale is what makes AFM-based data storage so enticing.

Though the AFM holds the potential for atomic-level storage, any practical implementation of such a scheme is still far from reality. As in STM, a major obstacle is the need to move the tip at high speeds over a surface while 


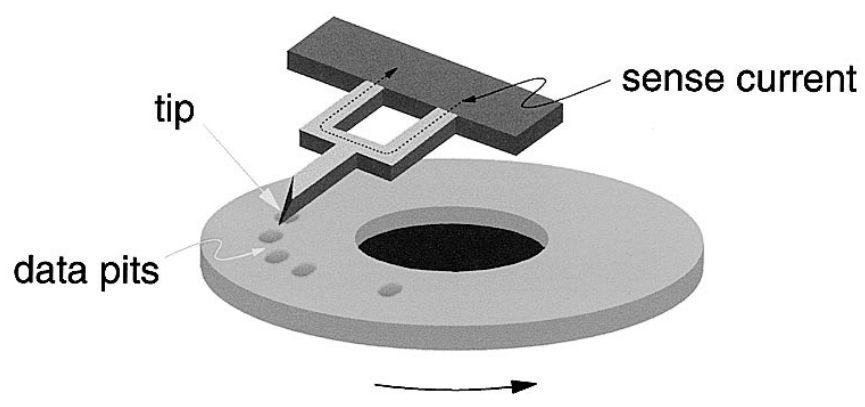

Fig. 3. Concept of topographic data storage using an AFM tip. The tip rides over the surface of the substrate, causing deflection of the cantilever as the tip moves over a topographic feature. The deflection is detected via a piezoresistive sensor.

controlling the tip-sample separation at the angstrom level. One can alternatively operate with the tip in contact, as suggested in Fig. 3. The advantage here is that, instead of trying to maintain the tip at a fixed height above the rapidly moving surface, following every hill and valley, the tip automatically follows the surface topography due to the compliance of the cantilever. (Of course the varying topography will affect the loading force, so that the feature heights must be kept below a reasonable limit.) By eliminating the need for an active servo, the potential for high data rate is increased. The tradeoff is that one has to deal with the tribology issues of sliding contact and its implications for reliability. Also, even at low loading forces, the peak stresses can be quite high if the tip is very sharp. The result is that the bit sizes achieved in contact mode tend to be far from the atomic scale. Nonetheless, the densities are still high enough to be of considerable interest, and contact-mode remains the basis for the most highly developed AFM storage approaches.

In the end, the chief advantage of the AFM approach to data storage may rest in its simplicity. Compared to a magnetic recording head, the tip is a very simple structure. It is reasonably straightforward, using conventional lithography and modern micromachining techniques to make tips that are sharp on the $100 \AA$ scale, if not the atomic scale. Indeed, the entire concept of AFM storage is in principle not very complicated: one has the media, a tip with some type of sensor, and the means to move one relative to the other. The technique could potentially be simple, compact, and inexpensive.

\section{Nontopographic Schemes for Probe-Based Data Storage}

The tip of the AFM can respond not only to topographic changes in the surface, but also to nontopographical ones, such as electrical or optical properties. An exhaustive review of AFM data storage efforts is beyond the scope of this paper. However, some examples of nontopographic approaches are described below to illustrate the range of possibilities. In most cases, the AFM cantilever is used primarily as a convenient way to allow tip-sample contact at low loading forces.

One promising scheme based on charge storage in nitrideoxide-semiconductor (NOS) structures has been demon- strated by Barrett and Quate [11]. The principle is similar to a flash memory, using the tip as a moving electrode. A voltage pulse on the tip causes charge to tunnel from a silicon substrate through an oxide barrier into a nitride film, where it is trapped. This causes a depletion region that can be detected as a change in capacitance. Using a conducting AFM tip operating in contact, Barrett and Quate were able to show highly reliable writing with marks as small as $700 \AA$. The technique has the advantage that it is electrical, so it is not limited by mechanical data rates. Though not strictly rewritable in the usual sense, it is reversible, with submillisecond erase times and up to $10^{6}$ read-write cycles expected [11], [12]. It is also not strictly necessary to operate in contact, though the loss of signal is precipitous as one introduces an air gap, due to the low dielectric constant of air. It remains to be seen whether signal-to-noise issues and media issues will permit higher densities. A more immediate difficulty encountered by Terris and Barrett is that of tip wear [13]. The nitride layer is very hard, and it is very effective at blunting the tip, especially as one goes toward reasonably high velocity between tip and sample. It may be that with advances in conducting diamond tips or carbon nanotube tips [14], this problem could be ameliorated. Depending on what the ultimate density limits of this technique turn out to be, this technique could merit more attention.

Another electrical approach, demonstrated by Kado and Tohda [15], uses a conducting tip to inject current into a chalcogenide film similar to that used in optical phasechange recording [16]. The medium heats up or otherwise is affected by the high current density or field and changes from an amorphous phase to a crystalline phase. As a result of the phase transformation, the properties of the medium change, in particular its resistivity. The marks are then read by measuring resistance between tip and substrate as the tip is scanned over the marks. In an impressive demonstration, marks on the order of $100 \AA$ were written and read back in this way. The technique requires a robust conducting tip and a relatively low tip-sample resistance, requirements that can be challenging when there is sliding contact [17]. Again, advances in conducting diamond tips or other coating technologies could alleviate this problem.

Nontopographic approaches based on near-field optical techniques have also been explored. The use of nearfield scanning optical microscopy (NSOM) has been demonstrated by Betzig et al. to perform reading and writing in magneto-optic materials [18]. In order to get around the problem of low photon flux through the nearfield apertures in traditional NSOM, Zenhausern, Martin, and Wickramasinghe have developed an apertureless technique dubbed SIAM for scanning intereferometric apertureless microscopy [19]. In this approach, a tip is used in place of an aperture. The tip acts as a localized scatterer of light, with its scattering properties modulated by the dipole-dipole interaction with the sample. A heterodyne detection scheme, in which the scattered light interferes with a reference beam, is used to increase the photon flux at the detector. Imaging on the $10-\AA$ scale has been 
demonstrated with this technique, and Martin et al. have used the approach to read out compact-disk-like patterns with a minimum feature size of $500 \AA$ [20]. An analysis of the signal-to-noise ratio suggests that data rates of 30 $\mathrm{MHz}$ or higher might be achievable. This approach has the advantage that it does not require contact between the tip and sample, though in that case, some means must be found to keep them in close proximity at high relative speeds.

One way to address this last issue is by adopting the type of air-bearing technology used in magnetic recording. This approach has been used to perform a type of nearfield optical recording using a special type of lens known as a solid immersion lens (SIL). The SIL is made of a high index material, which allows for a smaller focused spot within the lens [21]. By flying the lens close to the media, bits that are smaller than the optical diffraction limit have been written and read [22].

\section{AFM DATA STORAGE WITH TOPOGRAPHIC READBACK}

In the remainder of the paper, we will discuss in some detail one particular approach to AFM data storage, focusing on the work done in our own laboratory. The approach is that of mechanical readback of topographic features on a rotating disk with contact-mode AFM. We describe the advances in cantilever development to create highfrequency cantilevers with integrated piezoresistive sensors that allow high readback rates and compact implementation. A writing scheme based on heating of the tip is described, as well as an alternative means for stamping read-only disks with sub-1000 $\AA$ features. We also discuss some of the critical systems issues. In particular, we show that tracking on data on $1000 \AA$ wide tracks is possible. We discuss the reliability issue and show that for the favorable combination of a soft media and a hard tip, extended operation without any media wear is possible. Finally, we discuss some of the challenges, technical and otherwise, on the road ahead.

The basic concept of this scheme is shown in Fig. 3. A disk containing topographic features rotates under the tip. As the features move under the tip, the tip rides down into the pit, causing deflection of the cantilever, which is detected by some sort of sensor. This very simple concept did not originate with the AFM: it hearkens back to the original phonograph. Other stylus-based schemes have also been developed, such as the RCA video disk [23]. What distinguishes the AFM approach in this case is the ability to microfabricate the very sharp tips and operate at very low forces, so that one can achieve very high density and high data rate.

\section{A. Cantilevers}

Clearly the cantilever and the sensor structures are at the heart of the technique. Making suitable micromechanical structures has been a major part of the effort in our laboratory. What makes these components a challenge is that they have to satisfy many conditions simultaneously. They must have high resonant frequency, because in this scheme the data rate is determined by the mechanical

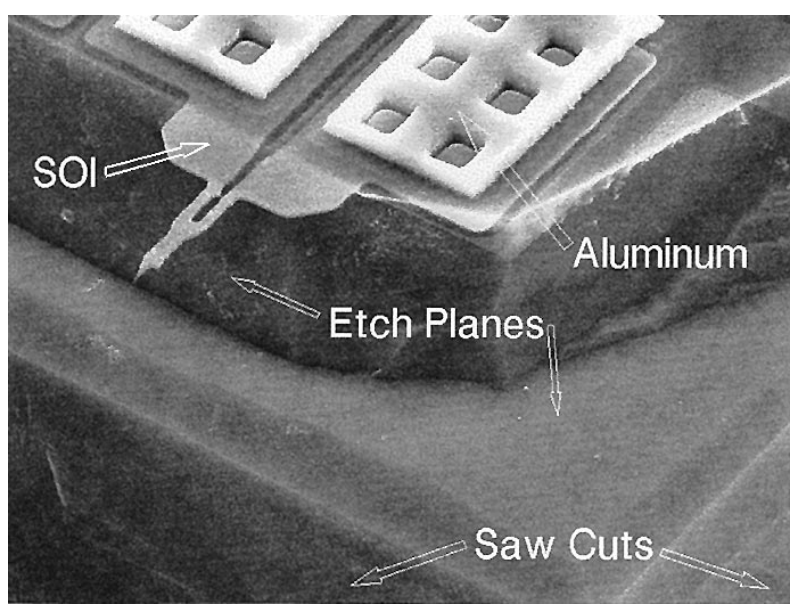

Fig. 4. Scanning electron micrograph of a $0.34 \mu \mathrm{m}$ thick, 12 $\mu \mathrm{m}$ long, high-frequency single-crystal silicon cantilever with an integrated piezoresistive sensor. The resonant frequency of this device is $2.4 \mathrm{MHz}$

response time. (Another way to achieve high data rates, by having multiple tips operating in parallel, will be described later.) High resonant frequency can be achieved by making the cantilevers stiff, but that is unacceptable, because low loading forces are required to prevent damage to the tip or media. The way to achieve both high resonant frequency $\omega$, and low stiffness $k$, is to make the moving mass $m$ small, as can be seen by the simple harmonic oscillator relation $m \cong k / \omega^{2}$. Thus, the cantilevers should be thin, narrow, and short. There must of course be a sharp tip on the end. Finally, there must be a way to sense the motion of the cantilever, and the sensitivity must be adequate to detect the small deflections in the bandwidth of interest.

The deflection sensor should ideally be integrated into the cantilever. Tortonese et al. have demonstrated the use of piezoresistive silicon cantilevers as integrated sensors [24]. We have taken this approach and further developed it to achieve high-frequency cantilevers.

Fig. 4 shows a scanning electron micrograph of one type of single-crystal silicon piezoresistive cantilever that we have developed [25]. It has a stiffness of about $1 \mathrm{~N} / \mathrm{m}$, a resonant frequency of $2.4 \mathrm{MHz}$, a length of $12 \mu \mathrm{m}$, and a thickness of $0.34 \mu \mathrm{m}$. An aluminum contact pad is used for injecting the current into the silicon cantilever, where it flows through a U-shaped silicon path and back to the other pad. The cantilever is made from a silicon-oninsulator (SOI) wafer and requires some novel processing steps. Typically, AFM cantilevers are made through a bulk release process in which the entire wafer thickness is etched away from the back. This allows the cantilevers to overhang the support, but it gives very poor control on the final length of the freestanding cantilever. For our short cantilevers, we needed to develop an alternative. The cantilever shown here was made with a front side release process, which gives excellent control on the length. One alternative that has recently been demonstrated is to do a backside release with a deep reactive ion etch through the thickness of the wafer, which also gives excellent control [26]. 
Incorporating piezoresistive sensors into such thin cantilevers is a significant challenge, since the current must be confined to one side of the neutral plane of the cantilever. Otherwise, the piezoresitive response due to the compressive stress on one side cancels the response due to the tensile stress on the other. To achieve this in our $0.34 \mu \mathrm{m}$ thick cantilever, we used a low-energy implant followed by a rapid thermal anneal [25], [27]. Further details of the fabrication process can be found in [25].

The above cantilever also used a novel type of in-plane tip. In this case, the tip sticks out like a spear from the cantilever. To access the surface of a sample with the in-plane tip, the entire support chip and cantilever are angled at a fairly steep attack angle, 30-45.$^{1}$ The advantage of the in-plane tip is that it adds essentially no mass to the cantilever and is very compatible with thin levers. In contrast, the mass of a more traditional tip can load down the cantilever considerably and reduce its resonant frequency, especially if one goes to even smaller cantilevers. Also, the more traditional out-of-plane tips are made through a subtractive process, so that one starts with a silicon layer as thick as the tip is high [24]. To end up with a thin lever, one needs extremely good tolerances to achieve the desired thickness. With the in-plane tip, the initial and final thicknesses of the silicon layer are the same, giving very good thickness control.

The in-plane tip is formed by the intersection of three planes. One plane of the tip is that of the cantilever, one is a plane perpendicular to the cantilever defined through a dry etch, and the third is a $\{111\}$ crystallographic plane defined in an anisotropic wet etch. Because the etch stops preferentially on $\{111\}$ planes, the process is very robust, and good tip uniformity can be achieved over the wafer. As evident in the scanning electron micrograph in Fig. 5, the tips can have a radius of curvature down to $40 \AA$.

The piezoresistive sensor on the cantilever has a displacement resolution down to $10^{-3} \AA / \mathrm{Hz}^{1 / 2}$, as shown in the noise spectrum in Fig. 6. The baseline noise is close to the intrinsic Johnson noise of the piezoresistor. The integrated noise in a 3-MHz bandwidth would be about $2 \AA$. In actuality, the integrated noise is typically higher (4-6 $\AA$ ), because of excess $1 / f$ noise. However, for features that are $200 \AA$ high, the minimum feature height of our samples, the ratio of signal to sensor noise is at least $30 \mathrm{~dB}$ in a $3-\mathrm{MHz}$ bandwidth, which is more than adequate. Fig. 6 shows a noise peak at $3 \mathrm{MHz}$, which is the resonant frequency of our cantilever. This peak is due to the thermal-mechanical motion of the cantilever. It is insignificant for the datastorage application, as the integrated noise is negligible, but it does represent the first time to our knowledge that a piezoresistive cantilever has been sensitive enough to detect its own thermal noise in air.

\footnotetext{
${ }^{1}$ One should note that the effective stiffness of the cantilever changes with the attack angle of the cantilever. If the cantilever is at an angle $\theta$ with respect to the horizontal axis, then an analysis of the forces reveals that the effective spring constant $\partial F_{z} / \partial z=k_{0} / \cos ^{2} \theta$, where $z$ is the vertical axis, and $k_{0}$ is the spring constant as usually defined.
}

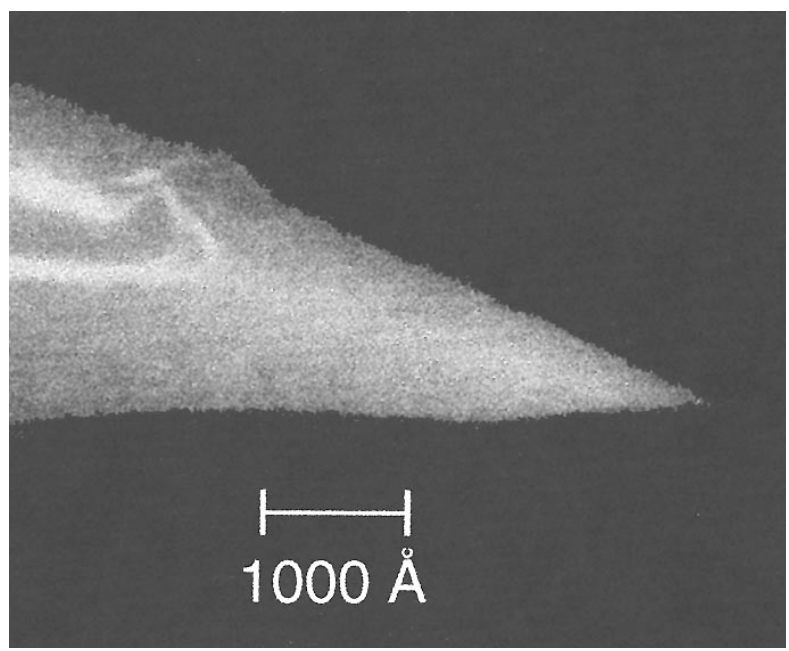

Fig. 5. Scanning electron micrograph showing a close up of the in-plane tip. The radius is estimated to be $40 \AA$.

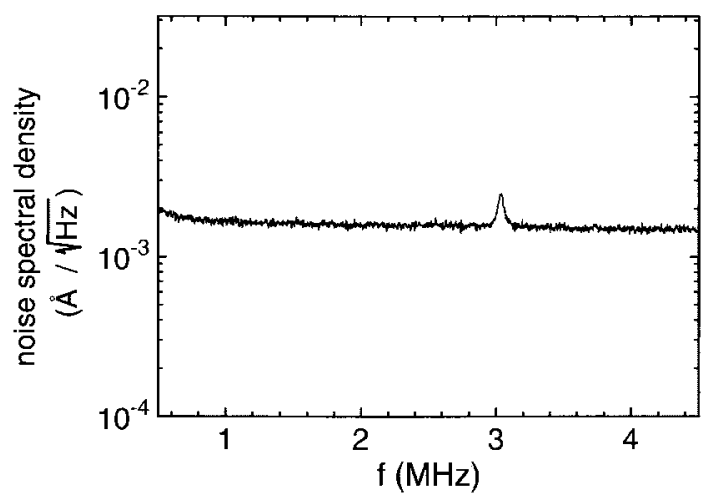

Fig. 6. Noise spectral density of the piezoresistive cantilever showing its displacement resolution. The baseline noise of 1.3 $\times 10^{-3} \AA / \mathrm{Hz}^{1 / 2}$ is due to the Johnson noise of the resistor. The thermal-mechanical motion of the cantilever at its resonant frequency of $3 \mathrm{MHz}$ is clearly visible.

We have made even higher frequency piezoresistive cantilevers than described above. One example is shown in Fig. 7; it is $12 \mu \mathrm{m}$ long and has a $4.6-\mathrm{MHz}$ resonant frequency. We have also made an $8 \mu \mathrm{m}$ long version with a $9-\mathrm{MHz}$ resonant frequency. These should support data rates of at least $10 \mathrm{Mbit} / \mathrm{s}$, as discussed below.

\section{B. Write-Once and Read-Only Schemes}

The above cantilevers are made for mechanical readback of topographic information. The problem remains of how to write the data on the disk. One scheme that we have pursued for some time is that of thermomechanical writing [28]. In this technique, the medium is a polymeric material such as polycarbonate or polymethyl methacrylate (PMMA) in either bulk or thin-film [29] form. The tip is heated by some means, causing heating and softening of the medium where the tip is in contact with it. The local pressure from the tip then creates an indentation. In the original implementation [28], the tip heating was done via an external laser focused on the tip. With this approach, it was possible to achieve 


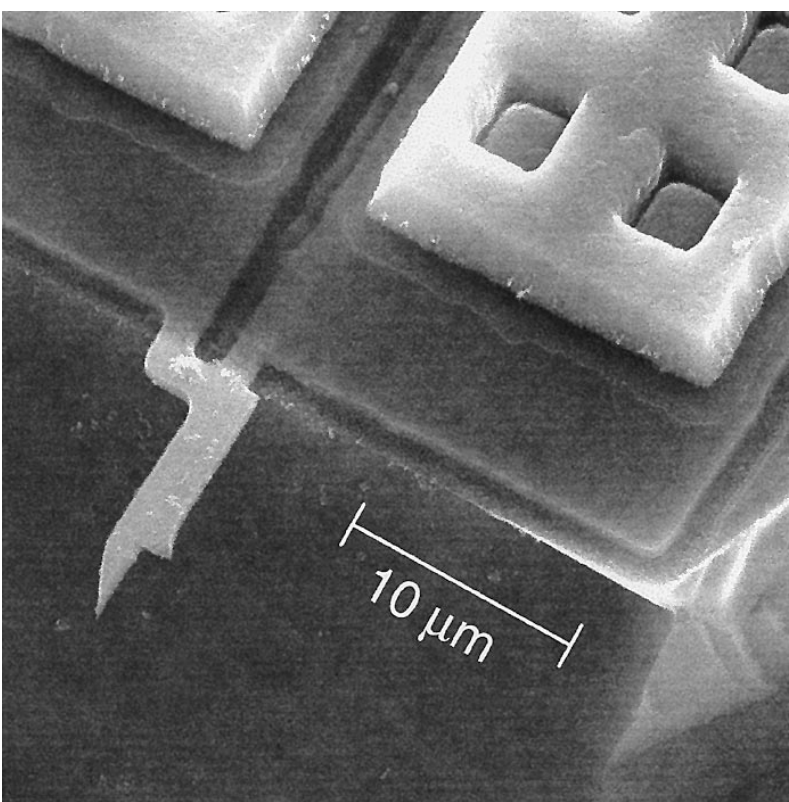

Fig. 7. Scanning electron micrograph of $12 \mu \mathrm{m}$ long cantilever with $4.6-\mathrm{MHz}$ frequency. The legs of this device are roughly 0.5 $\mu \mathrm{m}$ wide.

writing at repetition rates up to roughly $100 \mathrm{kHz}$ and produce data tracks that are $1500 \AA$ across.

Just as it is desirable to integrate the sensor onto the cantilever, it is likewise desirable to integrate a means to heat the tip and thereby eliminate the external laser. Ideally, it should be possible to read and write with a single cantilever by applying the appropriate electrical signals. Such a device could be extremely simple and compact, consisting only of a small micromachined element, an actuator, and a rotating disk. To achieve integrated heating, we have made electrical heaters near the tip [25], [30]. The heaters are produced by doping single crystal silicon, in a process similar to that used for the piezoresistors.

An integrated read-write device is shown in Fig. 8 [25]. The outer two legs of the four-legged device are used to inject current into a heater region defined near the tip. The legs are heavily doped to be low resistivity, except in the region near the tip, where lighter doping defines the tip heater. The inner two legs provide the current path for the piezoresistor. Again, the cantilever features an in-plane tip, and has an estimated resonant frequency of about $2 \mathrm{MHz}$.

Fig. 9(a) shows an AFM micrograph of data tracks written with the cantilever shown in Fig. 8. In addition, the readback waveform from the cantilever reading the data is shown in Fig. 9(b). This represents the first time that thermomechanical writing and readback have been performed with the same cantilever. While there was a certain amount of crosstalk due to the temperature sensitivity of the piezoresistor, it was relatively straightforward to compensate for this effect. The test was done on a rotating air-bearing spindle-based tester described in more detail below.

The marks on the upper track of Fig. 9(a) are roughly $1200 \AA$ across and represent a section of a pseudorandom

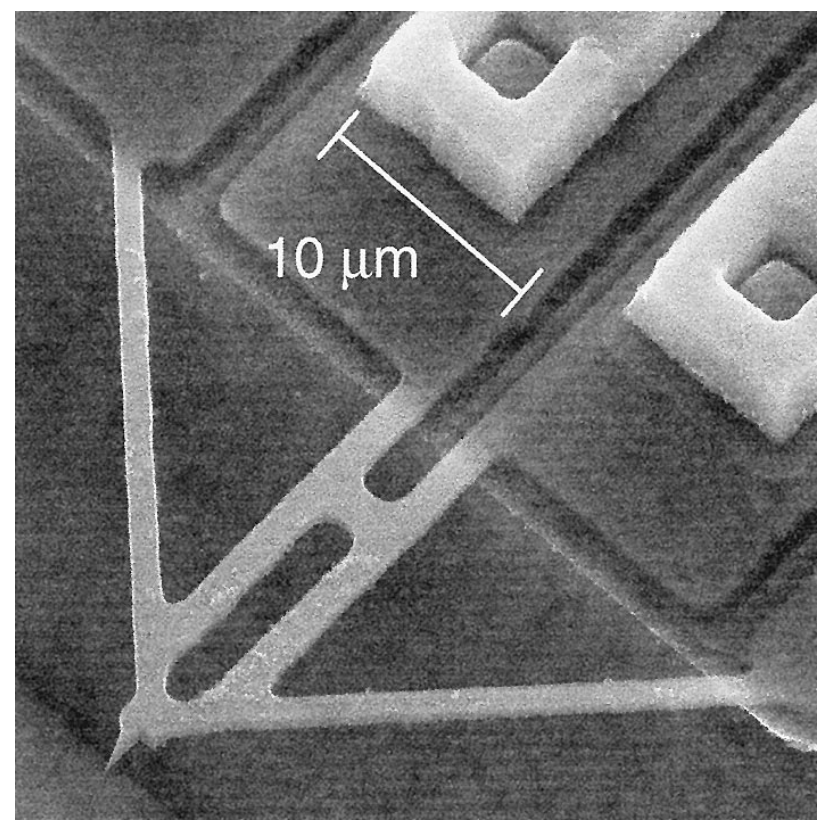

Fig. 8. Scanning electron micrograph of a $19 \mu \mathrm{m}$ long piezoresistive cantilever with a separate resistive heater for writing.

127-bit sequence of ones and zeros. (A one is represented by the presence of a pit, and a zero by its absence.) The data were written with a standard $(2,7)$ run-length limited code [31], meaning that there are between two and seven zeros between each one. The clock cycle was 30 $\mu \mathrm{s}$, corresponding to a data rate of $50 \mathrm{kbit} / \mathrm{s}$. Readback was performed at the same speed. The writing speed is limited by the thermal time constant, in particular the time necessary to cool the tip before the next mark can be written. For these levers, the single shot cooling time was measured to be about $4 \mu \mathrm{s}$. For a long train of pulses, heat builds up in the legs, causing the effective cooling rate to be reduced, reducing the maximum writing speed to roughly $50 \mathrm{kbit} / \mathrm{s}$.

To improve the thermal time constant, it is necessary to reduce the thermal mass of the region that is heated. For the laser-heated tip, the size of this region is determined by the diffraction-limited spot size, which can easily be $1 \mu \mathrm{m}$ or less. Cantilevers with micrometer-scale integrated heaters for optimized thermal response time have been fabricated by Chui et al. [30]. They have a geometric constriction near the tip to reduce the thermal mass of the heated region, as well as wider legs for better heat conduction to the cantilever support. The single-shot thermal time constant is only $1 \mu \mathrm{s}$ and can support writing at speeds up to $200 \mathrm{kbit} / \mathrm{s}$. This results in a write speed comparable to or greater than the laser-heated tip, with the benefit of greatly simplified implementation. Such high-speed heaters have not yet been combined with the piezoresistive sensors, however.

It should be noted that Hosaka and coworkers have made an impressive demonstration of a related form of writing that does not require the use of a heater [32]. In their scheme, the writing is purely mechanical. The cantilever support is mounted on a piezoelectric disk, which is pulsed to momentarily increase the loading force on the tip. In 


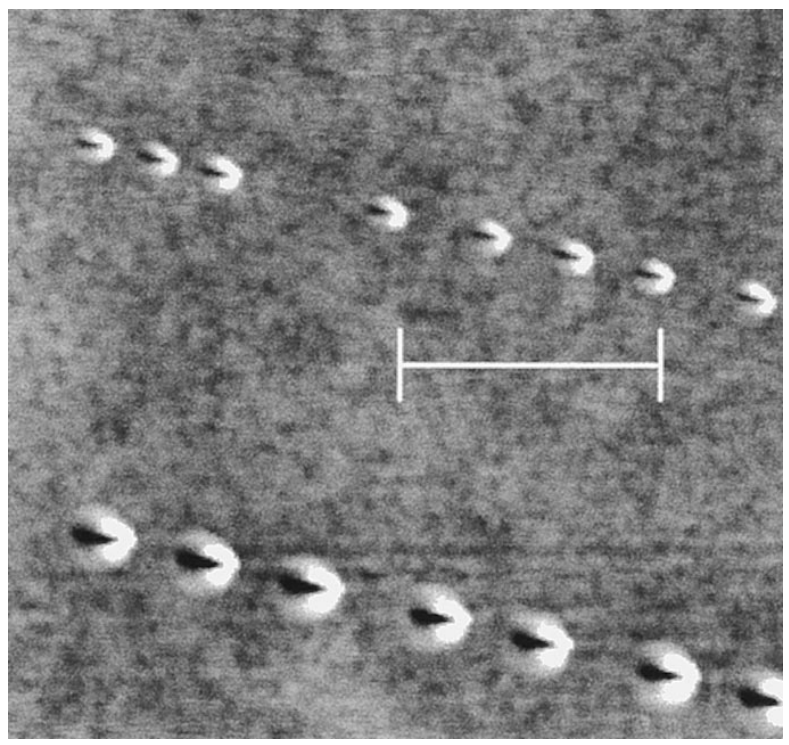

(a)

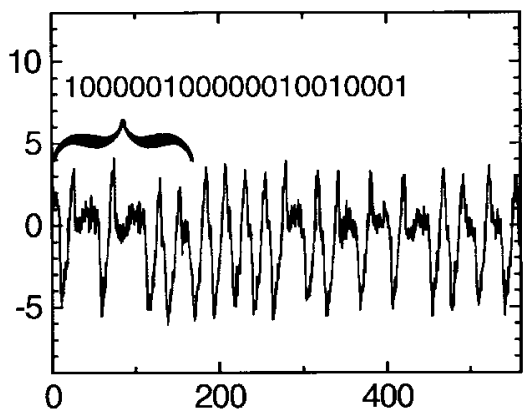

(b)

Fig. 9. (a) Atomic force micrograph of data tracks composed of $200 \AA$ high marks written thermomechanically with the integrated read-write device shown in Fig. 10. The scale bar is $1 \mu \mathrm{m}$ and (b) output waveform from the same device, reading at the same rate $(50 \mathrm{kbit} / \mathrm{s})$. Inset shows a portion of the rate $1 / 2(2,7)$ coding.

this way, the increased loading force causes a small pit to be made in the polycarbonate. Marks as small as $200 \AA$ across have been written in this way, leading to a density of 1 Tbit/in ${ }^{2}$, among the highest of the AFM-based schemes. The marks have been read back on a rotating disk at speeds up to $1 \mathrm{MHz}$ using low-mass cantilevers with an optical deflection sensor. The writing currently is slow, requiring 5-ms pulses, but that is almost certainly not the intrinsic limit.

Such topographic writing, whether thermally assisted or not, is a write-once technique, though bulk erasing may well be possible. (For example, surface smoothing using heating has recently been demonstrated [33].) For many applications, a write-once or read-only scheme may be adequate. In particular, the success of CD-ROM technology, for example, shows that there is a large market for inexpensive, read-only data storage. While higher density versions of CD-ROM, namely digital versatile disk (DVD)-ROM, are now available, the growth path is limited by the laws of optics and the wavelength of light. Tip-based techniques offer one means to extend the concept of readback of replicated topographic disks to even higher densities. One

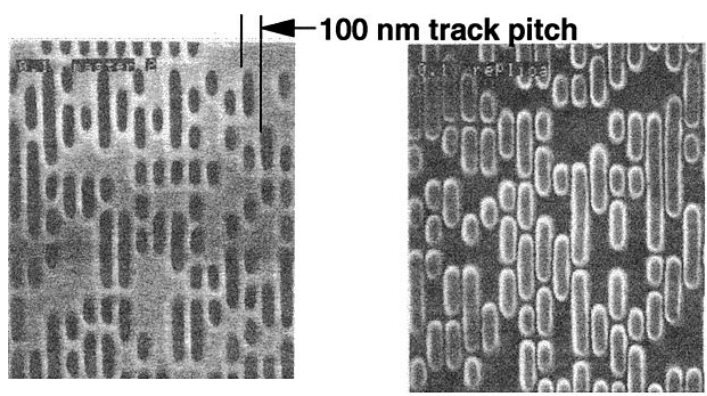

(a)

(b)

Fig. 10. AFM images of (a) the e-beam written master and (b) the replica of features written on a track pitch of $1000 \AA$.

key factor is whether the features can be fabricated and reliably replicated at the nanometer scale.

Numerous approaches to replication of small features have been demonstrated recently, such as imprint lithography by Chou et al. [34] and pattern transfer of selfassembled monolayers by the Whitesides group [35]. For our application, we have used an approach based on a photopolymer technique [36]. First, a master disk was produced with the necessary small data features using conventional electron-beam lithography. The substrate was a silicon wafer with a layer of silicon oxide on top. The pattern was written directly into a PMMA resist, and then a reactive ion etch was used to transfer the pattern into the silicon oxide. A thin layer of amorphous TbFeCo was then sputtered onto the substrate to form a release layer. A small amount of the liquid photopolymer consisting of a low-viscosity acrylate was placed on the master. A thin transparent substrate, typically a glass coupon, was then laid over the drop, causing it to flow over the area of the master. UV light was applied to cross link the photopolymer, turning it into a solid, hard layer. The glass substrate was then separated from the master, leaving the polymer replica bonded to the glass. The master could then be reused for making another replica.

It was immediately evident that the replication process does an excellent job of reproducing the topography of the master, even for feature sizes below $500 \AA$. Fig. 10 shows AFM micrographs of the master and the replica, showing excellent fidelity of the pits, which have $500-\AA$ minimum dimension. The rms roughness of the replica is only $2-5$ $\AA$, comparable to the master. It is also possible to make replicas of replicas in order to get back to the pit geometry and get a more direct comparison. The effective areal density in Fig. 10 is $65 \mathrm{~Gb} / \mathrm{in}^{2}$. Based on what we have seen, we believe that it is possible to replicate even smaller features and increase the density by another factor of four or so. Indeed, Krauss and Chou have recently demonstrated a form of imprint lithography capable of transferring patterns at densities up to $400 \mathrm{Gbit} \mathrm{in}^{2}$ [37].

\section{System Setup}

The basic cantilever technology and writing/replication means have been described above. In order to investigate 


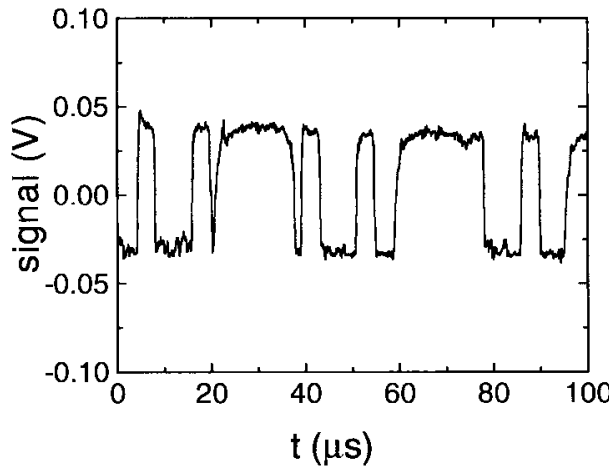

(a)

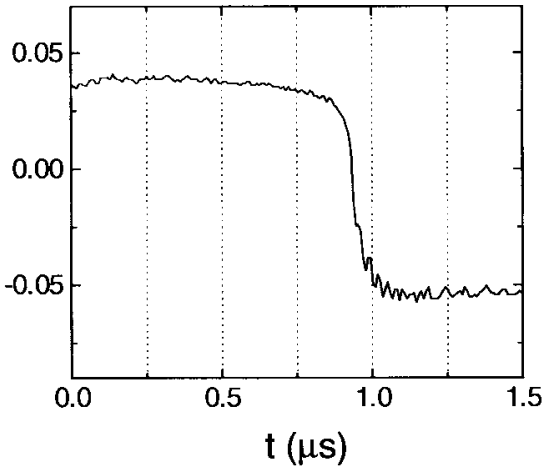

(b)

Fig. 11. (a) Output waveform from the 4.6-MHz device of Fig. 7 in response to $1100-\AA$ high features moving at $1 \mathrm{~m} / \mathrm{s}$ on a spinning disk and (b) blow up of one section of the waveform showing a $90 \mathrm{~ns}$ mechanical response.

real systems issues, such as data rate, error rate, timing jitter, track servoing, and long-term reliability, we have operated the cantilevers on a test stand capable of moving the media at high speeds under the tip. The tester is built around an air-bearing spindle that provides sample rotation. One advantage of rotary motion (as opposed to $x-y$ raster scanning), is that high linear velocities are easily achieved. Second, it is easy to cover a large area, and finally, one avoids the hysteresis effects and vibrations associated with a raster scan. The air-bearing spindles that we used have excellent stability and low run out.

With the ability to spin the sample rapidly, it is possible to determine the maximum speed at which the tip can track the surface. For this particular test, we did not have any load control operating. We simply operated open loop, letting the tip touch at the high points of the sample. We spun at 3600 $\mathrm{r} / \mathrm{min}$, giving a velocity at a radius of $2.5 \mathrm{~mm}$ of about $1 \mathrm{~m} / \mathrm{s}$. The sample in this case was a silicon calibration sample, which had a pattern of $1100 \AA$ deep features etched into it.

A section of the readback waveform is shown in Fig. 11, along with a high-resolution section showing the mechanical step response [25]. These data were taken with a cantilever of the type shown in Fig. 7. We observed a $10-90 \%$ fall time of roughly $90 \mathrm{~ns}$, which is sufficient to support a readback rate of at least $10 \mathrm{Mbit} / \mathrm{s}$. This is roughly equivalent to the peak data rate for the DVD.

In general operation, the cantilever is allowed to bend in order to follow small-scale variations in sample topography. A vertical servo is used, however, to maintain generally constant loading force around the circumference of the disk. This servo corrects for the vertical run out caused by sample tilt and only needs to operate at relatively low frequencies, well below the data rate. We have adapted the two-dimensional "fine" actuator from a CD player to act as our tip holder and positioner. The electromagnetically driven actuator has a range of hundreds of microns in both the vertical and cross-track directions. Using the signal from the piezoresistive cantilever as the error signal, a closedloop servo bandwidth of nearly $1000 \mathrm{~Hz}$ was achieved to control the vertical load on the cantilever [38]. Thermal and other drifts in the piezoresistor output will give rise to variations in loading force. To handle these variations during long-term testing, the tip is automatically lifted off the surface periodically and the error signal is rezeroed.

Equally important as servoing in the load direction is servoing in the cross-track direction. Staying on the data at track pitches on the $1000-\AA$ scale is essential to any type of tip-based data storage, or for that matter any high areal density storage scheme. The key to tracking is to generate a suitable error signal that will give information as to when the tip is on track, and if not, which direction it needs to go. In magnetic recording, one makes use of the fact that as the head moves off the track, there is a monotonic decrease in signal amplitude over some range governed by the finite lateral extent of the head and track. This allows one to use offset servo marks to give information as to where the head is relative to a given track. This basic approach of using offset servo marks for tracking has been demonstrated with the AFM, but it presented a number of challenges [39]. The main one is that the tip is narrower than the data being read, so that the linear regime of signal amplitude versus off-track position is very small.

A number of tracking schemes have been investigated as alternatives to using offset servo marks. One of these makes use of the lateral forces exerted on the tip as it rides up the sidewall of a pit. To measure such forces, a micromachined bidirectional force sensor capable of measuring both vertical and lateral forces was fabricated by Chui et al. [40]. A scanning electron micrograph of the sensor is shown in Fig. 12. Four high aspect-ratio vertical ribs provide the lateral compliance, with the triangular cantilever providing the vertical compliance. Both the ribs and the cantilever are piezoresistive, so that bidirectional sensing can be performed with minimal crosstalk. A completely different approach has been pursued by Nakamura and coworkers, who have described the use of an optical tracking scheme to achieve a $2000-\AA$ track pitch [41]. Finally, we have recently pursued a technique based on timing and have demonstrated the ability to stay on a given data track to within a few hundred angstroms with a bandwidth of a few hundred Hertz [38]. 


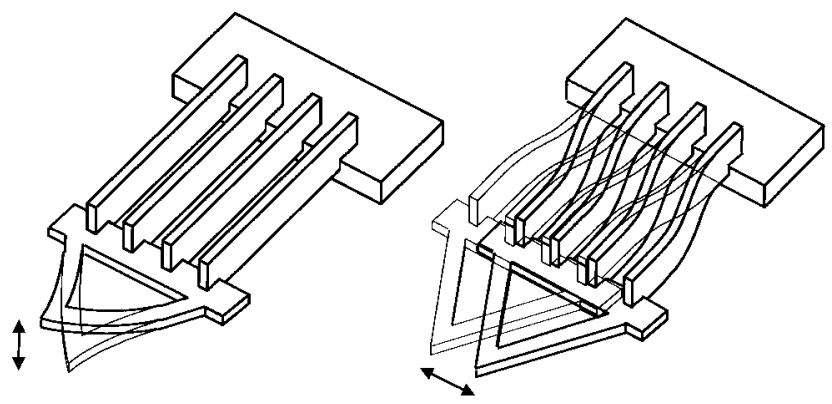

(a)

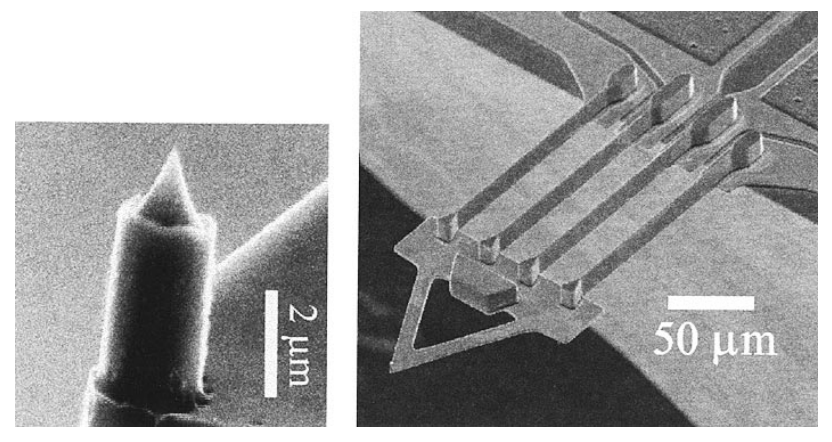

(b)

Fig. 12. (a) Bidirectional force sensor to measure the lateral and vertical forces independently and (b) single-crystal silicon implementation of the sensor with an integrated tip. The inner two ribs have piezoresistors implanted along the sidewalls to perform the lateral sensing, while the outer two ribs provide a current path to the V-shaped piezoresistive cantilever used to sense vertical motion. By sensing the lateral force on the tip and maintaining it at zero, it is possible to keep the tip centered in a groove. (Courtesy of B. Chui and T. Kenny, Stanford University.)

In the timing-based approach, the tip's radial position is obtained by measuring the time interval between special timing marks placed around the disk. The timing marks consist of groups of three lines, or "triplets," 1024 in all. These are made using the electron-beam mastering and replication process described above. As shown in Fig. 13(a), the center line of the triplet is in the radial direction, while the outer two lines are at a $22.5^{\circ}$ angle to it. The lines are approximately $30 \mu \mathrm{m}$ long, $1000 \AA$ wide, and $600 \AA$ high. Measuring the timing between the outer two lines gives a signal that is linear in the radial direction (the center line adds some robustness against errors). This is compared to a desired set point to generate a position error signal used to drive the tracking servo.

Data sectors were also written on the disk between the timing lines, as shown in Fig. 13(a) and (b). The data consist of circular marks, 1000 or $2000 \AA$ in diameter on up to $4000 \AA$ center-to-center spacing. Shown in Fig. 14(a) are waveforms from the four locations around the disk, showing the signal from the $1000 \AA$ marks and also the timing mark signals. The spacing between the timing marks, as well as the length of the data burst, demonstrate unambiguously that the tip is indeed staying on track around the complete disk. Shown in Fig. 14(b) is an expanded plot of the signal from one of the 1000- $\AA$ burst sections. Note that the peakto-peak signal amplitude for the 2000 - $\AA$ center-to-center

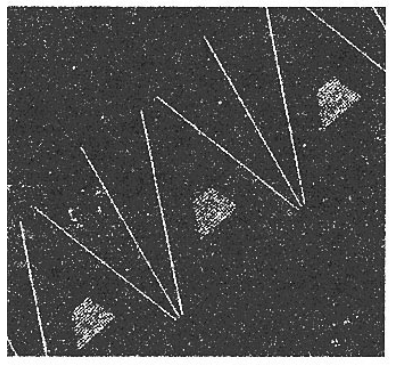

(a)

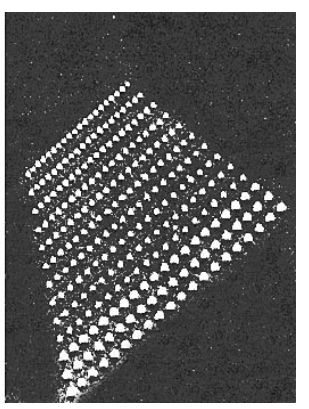

(b)
Fig. 13. (a) AFM image of timing lines used to perform track servo. (b) Between the lines are data bursts of various lengths.

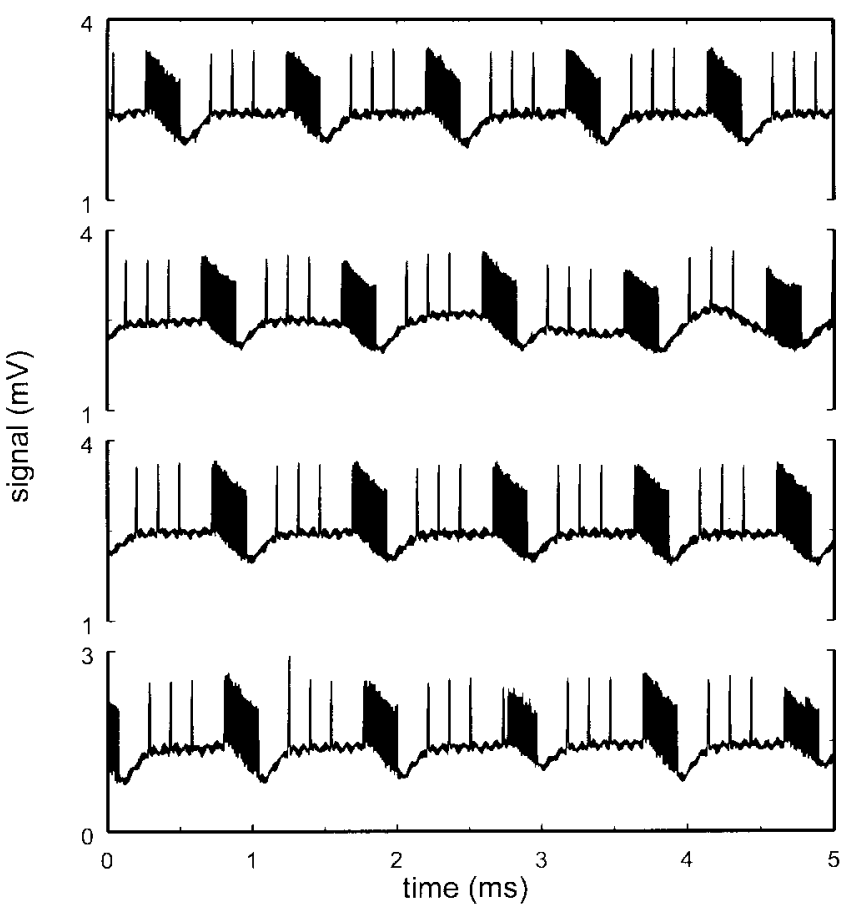

(a)

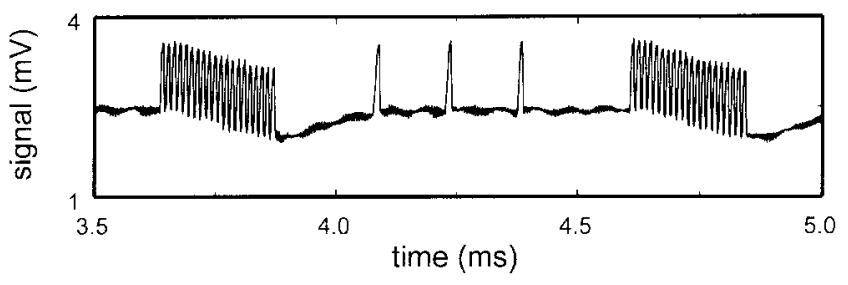

(b)

Fig. 14. Waveforms from four locations around the circumference of the disk, showing the ability of the track servo to maintain the tip on a given track of $1000 \AA$ marks. The shift in the baseline occurring after the data bursts is due to the operation of the load servo trying to keep the dc level constant.

spaced marks is identical to that of the isolated timing marks, demonstrating the ability of the in-plane tip to resolve such features.

The track error signal (TES) has been measured to characterize the performance of the tracking quantitatively. A histogram of the TES, as determined by the time interval measurement, is shown in Fig. 15. The disk was spinning 


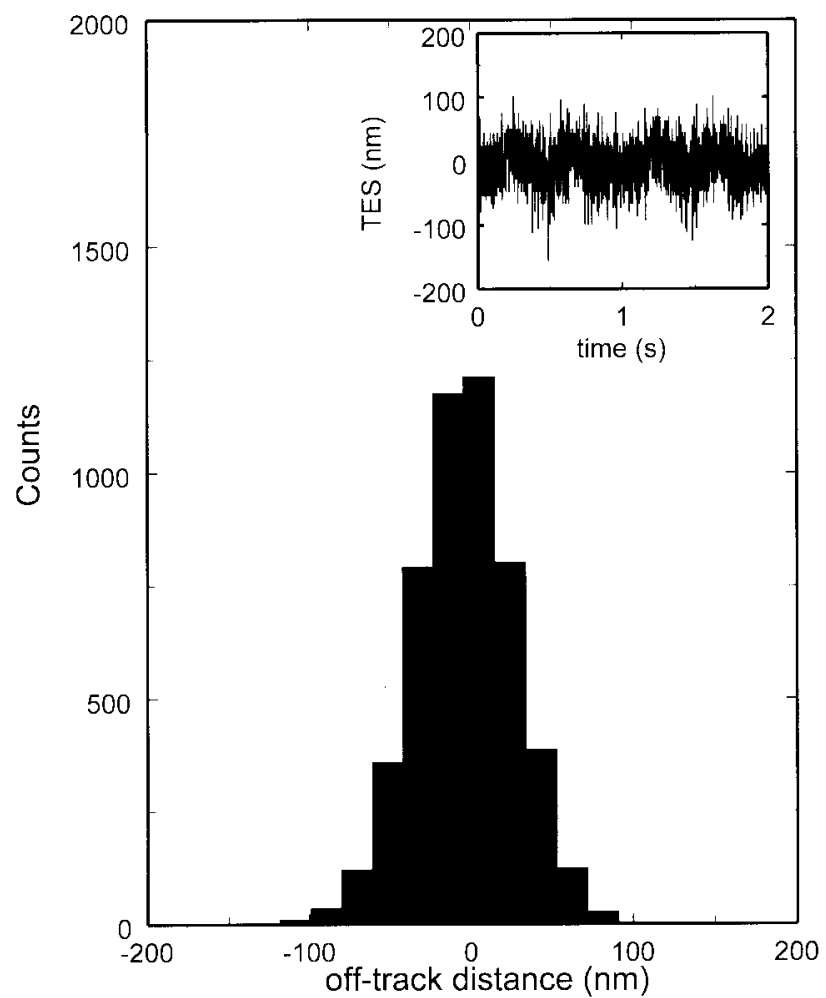

Fig. 15. Histogram of the timing error, showing a jitter corresponding to $31 \mathrm{~nm}(1 \sigma)$. The inset shows the track error signal as a function of time.

at $1 \mathrm{~Hz}$ at a track radius of $5 \mathrm{~mm}(30-\mathrm{mm} / \mathrm{s}$ linear velocity). The standard deviation $(1 \sigma)$ of the distribution was $310 \AA$. This implies that for a track pitch of 2000 $\AA$, the fraction of time that the tip is off track by 1000 $\AA$ or more is approximately $10^{-3}$. It should be pointed out that the electron-beam step size used in writing the timing lines was $125 \AA$, and thus timing differences arising from line jaggedness and field stitching are believed to contribute significantly to the perceived tracking error. Thus, improvements to electron-beam technology may be required if a track pitch below about $1000 \AA$ is desired. Even at the 2000- $\AA$ scale, though, the ability to show robust tracking with such a relatively simple servo is an encouraging sign and an important first step toward any real data-storage device.

\section{Wear and Reliability}

Clearly, one of the main concerns in this type of tipbased recording, or in any form of contact recording, is wear, both of the tip and of the sample. With the tip being in continuous contact with the spinning disk, it is expected that some wear will occur. However, by minimizing the loading force (typically $\leq 5 \times 10^{-8} \mathrm{~N}$ ) and using media that is softer than the tip, it is anticipated that tip wear will be reduced to an acceptable level.

In general, one expects a tradeoff to occur between density and wear. A smaller contact area between tip and media allows for higher density, but it also increases the loading stresses, making wear more probable. Lower loading force via a softer cantilever can reduce the stresses but tends to require lower frequency cantilevers and thus lower data rate, as well. A simple Hertzian analysis of a hard, spherical tip on a softer, flat media predicts that for the cantilever stiffnesses and loading forces at which we are operating, we are probably close to the onset of media wear at our densities of $65 \mathrm{~Gb} / \mathrm{in}^{2}\left(10 \mathrm{Gbit} / \mathrm{cm}^{2}\right)$ [25]. Thus, it is important to address this issue through testing.

To address the wear issue, tests have been made operating with the tip and media in contact for extended periods of time. The tests made use of the load servo to maintain the tip loading force and the tracking servo to maintain the tip on a given track. In one particular test, a cantilever with a spring constant of $0.5 \mathrm{~N} / \mathrm{m}$ was used, with an estimated average loading force below $5 \times 10^{-8} \mathrm{~N}$, and a $30 \mathrm{~mm} / \mathrm{s}$ disk velocity. (Due to the use of the angled cantilever at $45^{\circ}$, the effective spring constant was actually about 1 $\mathrm{N} / \mathrm{m}$.) The test was run for $148 \mathrm{~h}$, during which time the readback signal was monitored. The tip and sample were then characterized at the end of the test.

The test results can be summarized as follows. First, the device continued to operate reliably with good signalto-noise ratio throughout the entire period of the test. Indeed, as shown in Fig. 16, the readback waveforms from the $2000 \AA$ bits (4000 $\AA$ center-to-center) were virtually unchanged during a 142-h period of measurement starting at $6 \mathrm{~h}$ into the test. Second, there was some wearing of the tip, as evidenced by the fact that by the end of the test, the readback signal from the closely spaced $1000-\AA$ bits no longer showed the full peak-to-peak modulation. The tip radius after testing, as measured by scanning electron microscopy and shown in Fig. 17, is estimated to be 500 $\AA$, consistent with the observed resolution. Finally, no indication of media wear was seen in AFM images of the media, two of which are shown in Fig. 18. This test represents a total tip travel distance of $16 \mathrm{~km}$, with each bit having been read over 500000 times.

Based on these results, we believe that the tip wear is highly nonlinear in time, with some initial breakage or wear in occurring fairly quickly, at which point the tip becomes much more stable. This is to be expected: as the tip becomes less sharp, the pressure is reduced, and at some point the peak stresses fall below the threshold for damage. It appears from the consistency of the waveforms in Fig. 16 that the tip was below, or at least not far above, this threshold. While more extensive testing and modeling is needed, these results already demonstrate at least the possibility of obtaining realistic tip and media life times for AFM-based data storage having $2000 \AA$ bits.

To make even sharper tips that are stable, and to make the reading of even smaller bits more reliable, it will be necessary to tailor the tip and sample materials. Already we have seen that the relative wear rates of the tip and sample can be controlled by varying their respective hardness. By reducing the bake time of the polymer, one can reduce its hardness, which resulted in media that led to less tip wear, but increased media wear. Alternatively, baking the sample longer led to a harder sample and resulted in a dramatic loss 


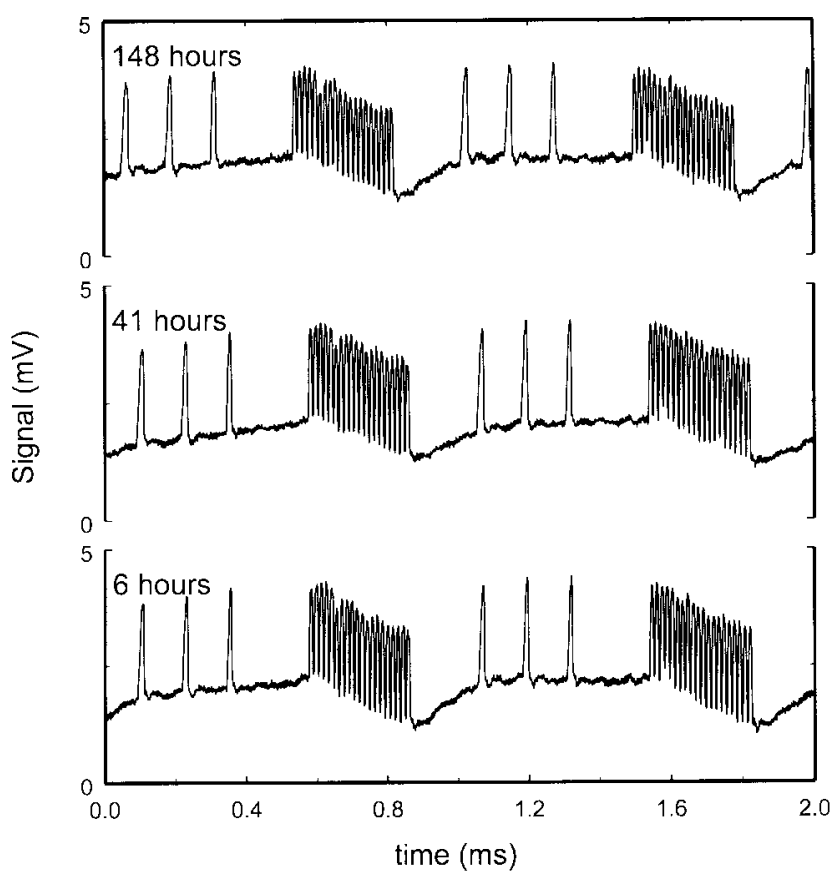

Fig. 16. Waveforms taken at various periods during an extended wear test lasting a total of $148 \mathrm{~h}$. The timing marks and the 2000- $\AA$ bits are clearly resolved throughout the length of the test. Each mark was read roughly half a million times during this test.

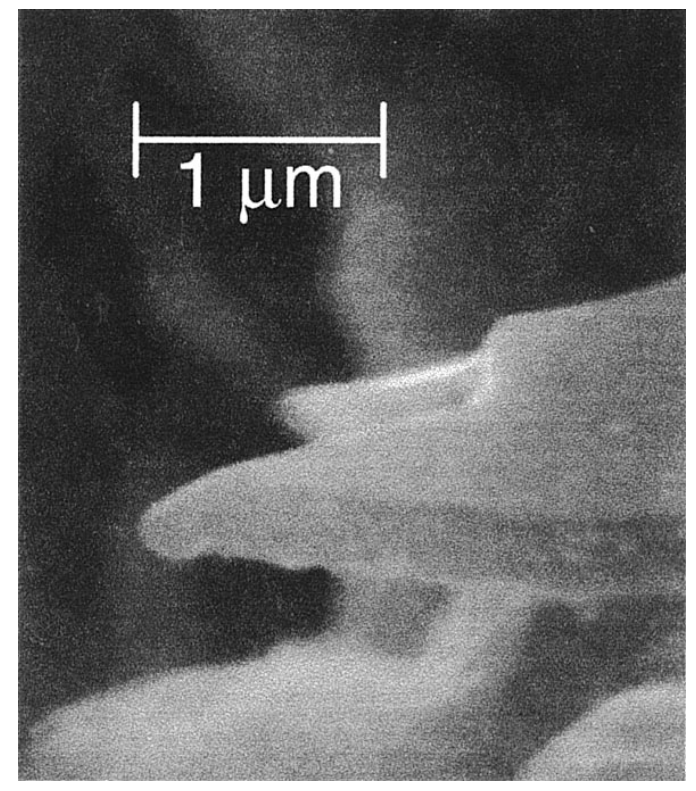

Fig. 17. Scanning electron micrograph of the tip after the 148-h wear test. The final tip diameter of roughly $1000 \AA$ is consistent with the resolution in the readback waveforms.

of tip resolution after a few hours. The optimum situation is likely to combine the hardest possible tip material, (e.g., diamond or silicon carbide) and a medium that is just hard enough to give acceptable wear rates during its lifetime. Ultimately, if it is not possible to maintain the desired tip sharpness for an acceptable period of time, it is not out of the question to build in redundant tips or the means to replace tips.

The reliability issue is probably the greatest technical challenge facing tip-based storage. Wear and contamination

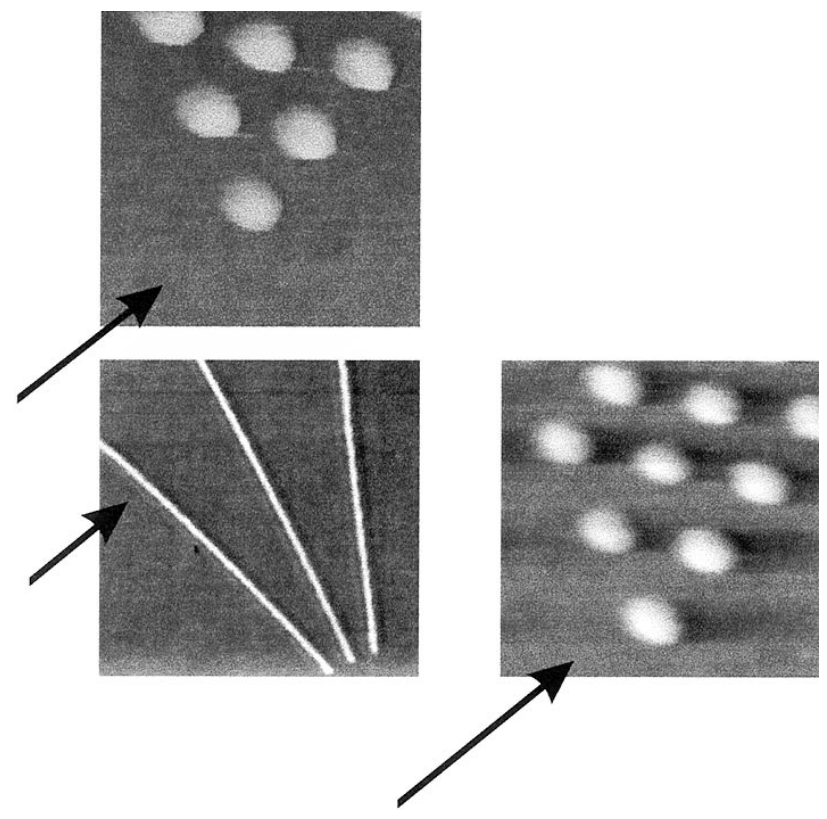

Fig. 18. AFM images of the sample after the extended wear test. Arrows mark the location and direction of the tip travel. On the scale of a few angstroms, no sample wear was detectable.

are potential problems that could ruin any tip-based device. The above results suggest that in a sufficiently clean and protected environment, continuous operation is possible at some level without losing the required tip sharpness or generating wear. On the other hand, in a sufficiently unfriendly environment, whether due to dirt, mechanical shock, vibrations, or temperature extremes, AFM storage devices can certainly be made to fail. The same statement could of course also be made about magnetic disk drives. The issue is how well can these problems be alleviated through proper engineering, and how reliable can the device be made under realistic operating conditions. More work is needed to make these assessments.

\section{MulTiPle TiP ARRAYS}

A complementary approach to achieving high data rates is to use parallel arrays of tips. In this approach, the data rate per tip can be relatively modest, but by having hundreds or thousands of tips in parallel, the effective data rate can be extremely high. Likewise, if there are enough tips so that each tip has to access only a fraction of the total storage area, then the access time will be reduced accordingly. Furthermore, if micromechanical actuators are used, the possibility exists for making the structure highly integrated, with electronics and mechanical motion on a single chip, and for making the entire structure highly shock resistant due to its low mass. Tip arrays for data storage are being explored at a number of laboratories, using tips operating in STM mode [42], field emission mode [43], or AFM mode [26], [29], [44].2

The rate of progress in this area has been quite rapid. Minne and coworkers in the Quate group at Stanford University have been at the forefront of fabricating and operat-

\footnotetext{
${ }^{2}$ TMS Technologies, Inc., Ithaca, NY, http://www.kionix.com.
} 


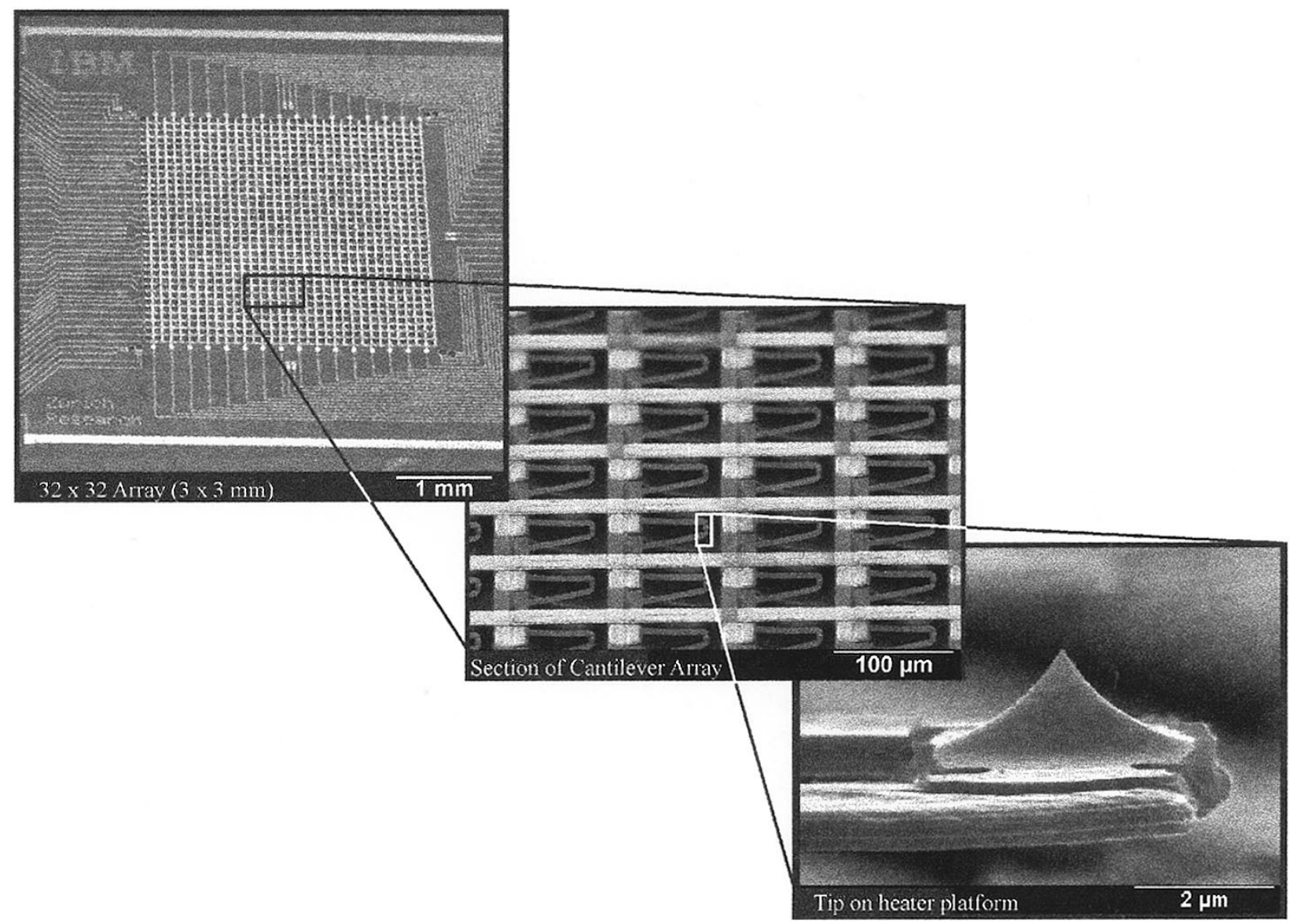

Fig. 19. Microfabricated $32 \times 32$ array of independently sensed silicon cantilevers for data storage applications (courtesy of P. Vettiger, IBM Research Division, Zurich Research Laboratory). Cantilevers can be addressed through crossing row and column interconnects, allowing for up to 32 cantilevers to be operated simultaneously. The $X Y$ pitch is $92 \times 92 \mu \mathrm{m}^{2}$.

ing AFM tip arrays, primarily for imaging and lithography applications [45], [46]. In an impressive demonstration, they have fabricated a one-dimensional array of 50 AFM cantilevers, each with an independent sensor and $z$ actuator, on a $200-\mu \mathrm{m}$ pitch. Separate electronics on a computercontrolled custom printed circuit card allow for servoing and data acquisition on multiple channels simultaneously.

At the IBM Zurich Research Laboratory, a twodimensional array for data storage applications has recently been fabricated [47]. The array, shown in Fig. 19, is a 32 $\times 32$ array of silicon cantilevers with integrated heaters. Single cantilevers with this design have already been used to demonstrate not just thermomechanical writing, but also thermal sensing of the pits [29]. Moreover, a thermal erasing scheme has also been demonstrated [33]. Using a thin film polymer media on a silicon substrate, uniform series of $400 \AA$ marks have been made [29], [33], suggesting the extendibility of this approach to densities as high as $400 \mathrm{Gbit} / \mathrm{in}^{2}$.

The tradeoffs in using tip arrays are mainly those of reliability, tolerances, and complexity. If the reliability is an issue with a single tip, it may be even more difficult to achieve with multiple tips, though one could build in some redundancy. There are also stringent requirements on the tolerances. For example, the loading force on the tip needs to be uniform on every tip to perhaps $10 \%$, which implies the loading distance might need to be set to within roughly $100 \AA$ A. This implies that either the cantilevers must be individually actuated, or else the tip heights and cantilever planarity must be uniform to that extent. Likewise, the tip positions in the lateral direction must be accurate to a similar extent. With this level of tolerance, even relatively small thermal expansions or other drifts might be enough to ruin the alignment. Some control of tip spacing in the lateral direction may therefore be necessary. This could be achieved to some extent with a skew servo, which changes the alignment angle of the tips relative to the data surface. Otherwise, individual actuation may be required. If each of the tips has to be actively servoed in $x$ and $z$, the increase in complexity is considerable.

Impressive work is underway in the area of fabricating arrays and demonstrating their operation. One can certainly envision that with a suitably large array, the desired level of performance could be achieved. It remains to be seen whether the challenges of the additional complexity can be overcome.

\section{PROSPECTS}

Tip-based data storage has the prospect for inexpensive and compact devices. Because it relies on tips that can be made very sharp, it has the potential for ultrahigh data density, with bit sizes from $1000 \AA$ down to the atomic 
scale $(<10 \AA)$. In all cases, the challenge remains that data storage is much more than just making small marks. Many other factors, such as reliability, access time, error rate, and cost, must also be considered.

In any nascent technology, it is also essential to consider where the existing technologies are headed. These technologies continue to progress, of course, while the alternative ones are still under development. In the case of magnetic recording, this progress has been nothing short of astonishing, and it makes it exceedingly difficult for any new potential technologies to compete. For example, it is reasonable to assume that in five years, a 3.5-in disk drive looking much like today's will be available with a capacity of roughly 100 Gbytes (nearly $10^{12}$ bits) at a cost of several hundred dollars. Any new technology must offer some competitive advantage if it is to be successful.

Perhaps the key to success for tip-based storage is in finding the right market niche. This approach to storage does have the advantage that it could be extremely inexpensive and, by virtue of its high density, could be compact, yet still have a high capacity. This makes it ideally suited to applications for personal storage, such as portable video, personal navigators, and the like. The potential market for such type of personal storage at this time is largely unclear, though as computing becomes more pervasive and portable, it could become significant.

Ultimately, the future of tip-based data storage depends on many factors besides its areal density. Factors such as cost, market demand, progress in competing technologies, and the emergence of novel applications, will all affect its viability. The lessons learned in exploring probe data storage, though, may well be useful regardless of the eventual outcome. And, as storage technology continues to move farther into the nanoscale regime, it may someday be necessary to call upon those techniques that can readily access this realm.

\section{ACKNOWLEDGMENT}

The authors wish to thank B. Chui and T. Kenny for their contributions to this project, P. Vettiger and G. Binnig for enlightening discussions and for sharing their recent results, and M. Best, S. Rishton, and B. McChesney for assistance in preparing the read-only samples and replicas.

\section{REFERENCES}

[1] E. Grochowski and R. F. Hoyt, "Future trends in hard disk drives," IEEE Trans. Magn., vol. 32, pp. 1850-1854, May 1996.

[2] C. Tsang, M. Pinarbasi, H. Santini, E. Marinero, P. Arnett, R. Olson, R. Hsiao, M. Williams, R. Payne, J. Moore, B. Gurney, T. Lin, and R. Fontana, " 12 Gbit/in ${ }^{2}$ recording demonstration with SV read heads and conventional narrow pole-tip write heads," IEEE Trans. Magn., vol. 35, pt. 1, pp. 689-694, Mar. 1999.

[3] G. Binnig, H. Rohrer, C. Gerber, and E. Weibel, " $7 \times 7$ reconstruction on $\mathrm{Si}(111)$ resolved in real space," Phys. Rev. Lett., vol. 50, no. 2, pp. 120-123, Jan. 1983.

[4] C. F. Quate, "Method and means for data storage using tunnel current data readout," U.S. Patent 4575 822, 1986.

[5] G. Binnig, C. F. Quate, and C. Gerber, "Atomic force microscope,” Phys. Rev. Lett., vol. 56, no. 9, pp. 930-933, Mar. 1986.
[6] U. Staufer, "Surface modification with a scanning proximity probe microscope," in Scanning Tunneling Microscopy II, 2nd ed., R. Wiesendanger and H.-J. Guntherodt, Eds. Berlin, Germany: Springer, 1995, pp. 273-302.

[7] C. F. Quate, "Manipulation and modification of nanometer scale objects with the STM," in Highlights in Condensed Matter Physics and Future Prospects, L. Esaki, Ed. New York: Plenum, 1991, pp. 573-630.

[8] D. M. Eigler and E. K. Schweizer, "Positioning single atoms with a scanning tunnelling microscope," Nature, vol. 344, no. 6266, pp. 524-526, Apr. 1990.

[9] H. J. Mamin, H. Birk, P. Wimmer, and D. Rugar, "High speed scanning tunneling microscopy: Principles and applications," $J$. Appl. Phys., vol. 75, no. 1, pp. 161-168, Jan. 1994.

[10] R. Erlandsson, L. Olsson, and P. Mårtensson, "Inequivalent atoms and imaging mechanisms in ac-mode atomic force microscopy of Si(111) 7×7," Phys. Rev. B, vol. 54, no. 12, pp. R8309-8312, Sept. 1996.

[11] R. Barrett and C. F. Quate, "Charge storage in nitride-oxidesilicon medium by scanning capacitance microscopy," J. Appl. Phys., vol. 70, no. 5, pp. 2725-2733, Sept. 1991.

[12] J. J. Chang, "Nonvolatile semiconductor memory devices," Proc. IEEE, vol. 64, pp. 1039-1059, July 1976.

[13] B. D. Terris and R. C. Barrett, "Data storage in NOS: Lifetime and carrier-to-noise measurements," IEEE Trans. Electron Devices, vol. 42, pp. 944-949, May 1995.

[14] H. Dai, J. H. Hafner, A. G. Rinzler, D. T. Colbert, and R. E. Smalley, "Nanotubes as nanoprobes in scanning probe microscopy," Nature, vol. 384, no. 6605, pp. 147-150, Nov. 1996.

[15] H. Kado and T. Tohda, "Nanometer-scale recording on chalcogenide films with an atomic force microscope," Appl. Phys. Lett., vol. 66, no. 22, pp. 2961-2962, May 1995.

[16] N. Yamada, E. Ohno, K. Nishiuchi, N. Akahira, and M. Takao, "Rapid-phase transitions of $\mathrm{GeTe}-\mathrm{SB}_{2} \mathrm{TE}_{3}$ pseudobinary amorphous thin films for an optical disk memory," J. Appl. Phys., vol. 69, no. 5, pp. 2849-2861, Mar. 1991

[17] R. E. Thomson and J. Moreland, "Development of highly conductive cantilevers for atomic force microscopy point contact measurements," J. Vac. Sci. Technol. B, vol. 13, no. 3, pp. 1123-1125, May/June 1995.

[18] E. Betzig, J. K. Trautman, R. Wolfe, E. M. Gyorgy, P. L. Finn, M. H. Kryder, and C. H. Chang, "Near-field magneto-optics and high density data storage," Appl. Phys. Lett., vol. 61, no. 2, pp. 142-144, July 1992.

[19] F. Zenhausern, Y. Martin, and H. K. Wickramasinghe, "Scanning interferometric apertureless microscopy: Optical imaging at 10 Angstrom resolution," Science, vol. 269, pp. 1083-1085, Aug. 1995.

[20] Y. Martin, S. Rishton, and H. K. Wickramasinghe, "Optical data storage read out at 256 Gbits/in²," Appl. Phys. Lett., vol. 71, no. 1, pp. 1-3, July 1997.

[21] S. M. Mansfield and G. S. Kino, "Solid immersion microscope," Appl. Phys. Lett., vol. 57, no. 24, pp. 2615-2616, Dec. 1990.

[22] B. D. Terris, H. J. Mamin, and D. Rugar, "Near-field optical data storage," Appl. Phys. Lett., vol. 68, no. 2, pp. 141-143, Jan. 1996

[23] J. R. Matey and J. Blanc, "Scanning capacitance microscopy," J. Appl. Phys., vol. 57, no. 5, pp. 1437-1444, Mar. 1985.

[24] M. Tortonese, R. C. Barrett, and C. F. Quate, "Atomic resolution with an atomic force microscope using piezoresistive detection," Appl. Phys. Lett., vol. 62, no. 8, pp. 834-836, Feb. 1993.

[25] R. P. Ried, H. J. Mamin, B. D. Terris, L.-S. Fan, and D. Rugar, " $6 \mathrm{MHz}, 2 \mathrm{~N} / \mathrm{m}$ piezoresistive atomic-force-microscope cantilevers with INCISIVE tips," IEEE J. Microelectromech. Syst., vol. 6, pp. 294-302, Dec. 1997.

[26] M. Lutwyche, C. Andreoli, G. Binnig, J. Brugger, U. Drechsler, W. Haeberle, H. Rohrer, H. Rothuizer, and P. Vettiger, "Microfabrication and parallel operation of $5 \times 5$ AFM cantilever arrays for data storage and imaging," presented at MEMS'98, Heildelberg, Germany, 1998.

[27] B. W. Chui, H. J. Mamin, B. D. Terris, T. D. Stowe, D. Rugar, and T. W. Kenny, "Low-stiffness silicon cantilevers for thermal writing and piezoresistive readback with the atomic force microscope," Appl. Phys. Lett., vol. 69, no. 18, pp. 2767-2769, Oct. 1996. 
[28] H. J. Mamin and D. Rugar, "Thermomechanical writing with an atomic force microscope tip," Appl. Phys. Lett., vol. 61, no. 8, pp. 1003-1005, Aug. 1992.

[29] P. Vettiger, J. Brugger, M. Despont, U. Drechsler, U. Dürig, W. Häberle, M. Lutwyche, H. Rothuizen, R. Stutz, W. Widmer, and G. Binnig, "Ultrahigh density, high data rate NEMS-based AFM data storage system," J. Microelectron. Eng., to be published.

[30] B. W. Chui, T. D. Stowe, Y. S. Ju, K. E. Goodson, T. W. Kenny, H. J. Mamin, B. D. Terris, R. P. Ried, and D. Rugar, "Low-stiffness silicon cantilevers with integrated heaters and piezoresisitve sensors for high-density AFM thermomechanical data storage," IEEE J. Microelectromech. Syst., vol. 7, pp. 69-78, Mar. 1998.

[31] C. D. Mee and E. D. Daniel, Magnetic Recording Technology, 2nd ed. New York: McGraw-Hill, 1996.

[32] S. Hosaka, H. Koyanagi, A. Kikukawa, M. Miyamoto, K. Nakamura, and K. Etoh, "Force modulation atomic force microscopy recording for ultrahigh density recording," J. Vac. Sci. Technol. $B$, vol. 15 , no. 4, pp. 788-792, July/Aug. 1997.

[33] G. Binnig, M. Despont, U. Drechsler, W. Häberle, M. Lutwyche, P. Vettiger, H. J. Mamin, B. W. Chui, and T. W. Kenny, "Ultra-high density AFM data storage with erase capability," Appl. Phys. Lett., vol. 74, no. 9, Mar. 1999.

[34] S. Y. Chou, P. R. Krauss, and P. J. Renstrom, "Imprint lithography with 25-nanometer resolution," Science, vol. 272, pp. 85-87, Apr. 1996.

[35] S. Palacin, P. C. Hidber, J.-P. Bourgoin, C. Miramond, C. Fermon, and G. M. Whitesides, "Patterning with magnetic materials on the micron scale," Chem. Mater., vol. 8, no. 6, pp. 1316-1325, June 1996.

[36] B. D. Terris, H. J. Mamin, M. E. Best, J. A. Logan, D. Rugar, and S. A. Rishton, "Nanoscale replication for scanning probe data storage," Appl. Phys. Lett., vol. 69, no. 27, pp. 4262-4264, Dec. 1996.

[37] P. R. Krauss and S. Y. Chou, "Nano compact disks with 400 Gbit/in ${ }^{2}$ storage density fabricated using nanoimprint lithography and read with proximal probe," Appl. Phys. Lett., vol. 71, no. 21, pp. 3174-3176, Nov. 1997.

[38] B. D. Terris, S. A. Rishton, H. J. Mamin, R. P. Ried, and D. Rugar, "Atomic force microscope based data storage: Track servo and wear study," Appl. Phys. A, vol. 66, pt. II, pp. S809-S813, 1998.

[39] H. J. Mamin, B. D. Terris, L. S. Fan, S. Hoen, R. C. Barrett, and D. Rugar, "High-density data storage using proximal probe techniques," IBM J. Res. Develop., vol. 39, no. 6, pp. 681-699, Nov. 1995.

[40] B. W. Chui, T. W. Kenny, H. J. Mamin, B. D. Terris, and D. Rugar, "Independent detection of vertical and lateral forces with a sidewall-implanted dual-axis piezoresistive cantilever," Appl. Phys. Lett., vol. 72, no. 11, pp. 1388-1390, Mar. 1998.

[41] K. Nakamura, H. Koyanagi, and S. Hosaka, "Narrow pitch tracking for AFM recording using optical head," Jpn. J. Appl. Phys. 1, vol. 37, no. 4B, pp. 2271-2273, Apr. 1998.

[42] G. K. Fedder, S. Santhanam, M. L. Reed, S. C. Eagle, D. F Guillou, M. S.-C. Lu, and L. R. Carley, "Laminated high-aspectratio microstructures in a conventional CMOS process," Sensors Actuators A, vol. 57, no. 2, pp. 103-110, Nov. 1997.

[43] G. Gibson, T. I. Kamins, M. S. Keshner, S. L. Naberhuis, C. M. Perlow, and C. C. Yang, "Ultrahigh density storage device," U.S. Patent 5 557 596, 1996.

[44] Nanochip, Inc., Hayward, CA, as described in C. Brown, "Microprobes promise a new memory option," EE Times, pp. 6-7, Jan. 12, 1998.

[45] C. F. Quate, "Scanning probes as a lithography tool for nanostructures," Surf. Sci., vol. 386, no. 1-3, pp. 259-264, Oct. 1997.

[46] S. C. Minne, G. Yaralioglu, S. R. Manalis, J. D. Adams, J. Zesch, A. Atalar, and C. F. Quate, "Automated parallel highspeed atomic force microscopy," Appl. Phys. Lett., vol. 72, no. 18, pp. 2340-2342, May 1998.

[47] M. Despont, J. Brugger, U. Dreschler, U. Durig, W. Haberle, M. Lutwyche, H. Roithuizen, R. Stutz, R. Widmer, H. Rohrer, G. K. Binnig, and P. Vettiger, "VLSI-NEMS chip for AFM data storage," in IEEE Int. Micro Electro Mechanical Systems Tech. Dig. 1999, Orlando, FL, pp. 564-569.

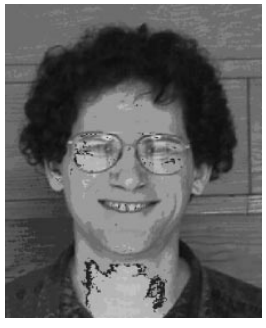

H. Jonathon Mamin received the B.S. degree in physics from Stanford University, Stanford, CA, in 1978 and the Ph.D. degree in physics from the University of California, Berkeley, in 1984

After receiving the Ph.D. degree, he remained at Berkeley as a Postdoctoral Fellow in the then emerging field of scanning tunneling microscopy. Since 1987, he has been a Research Staff Member at the Almaden Research Center, IBM Research Division, San Jose, CA. He has a long-standing interest in surface modification using scanning probes, as well as in micromechanics and ultrahigh density data storage.

Dr. Mamin is a member of the American Physical Society and the American Vacuum Society.

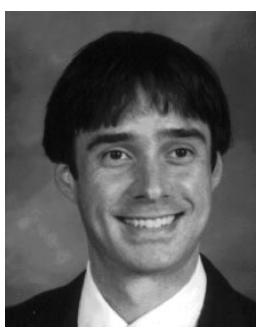

Robert P. Ried (Member, IEEE) received the B.S. degree in electrical engineering from Rice University, Houston, TX, in 1984, the M.Eng. degree from Cornell University, Ithaca, NY, in 1985, and the Ph.D. degree from the University of California, Berkeley, in 1994.

From 1985 to 1987, he was employed at AT\&T Bell Laboratories, Holmdel, NJ, as a designer of long-distance, fiber-optic transmissions systems. From 1995 to 1998 , he was a Postdoctoral Researcher at the Almaden Research Center, IBM Research. He is presently a Product Engineer at Input/Output, Inc., Stafford, TX. His MEMS projects have focused on micromachined microphones, scanning-probe data storage, and vibration sensors.

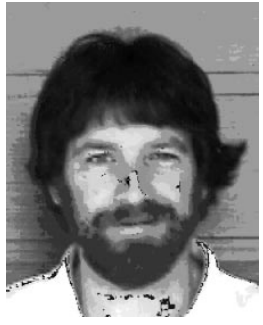

Bruce D. Terris received the B.S. degree in applied physics from Columbia University, New York, in 1979 and the M.S. and Ph.D. degrees in physics from the University of Illinois, UrbanaChampaign, in 1981 and 1983, respectively.

After receiving the Ph.D. degree, he was a Postdoctoral Fellow for two years at Argonne National Laboratory. In 1985, he joined IBM as a Research Staff Member at the Almaden Research Center, San Jose, CA, where he is currently the Manager of Exploratory Magnetic Media and Phenomena. His research interests have included thin film superconductivity and magnetism, contact electrification of insulators, and new types of scanning probe microscopes (STM, AFM, near-field optical, etc.). His current research is on patterning of magnetic structures for highdensity data recording.

Dr. Terris is a member of the American Physical Society.

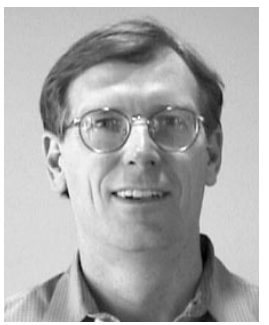

Daniel Rugar (Member, IEEE) received the B.A. degree in physics (magna cum laude) from Pomona College, Claremont, CA, in 1975 and the Ph.D. degree in applied physics from Stanford University, Stanford, CA, in 1982

From 1982 to 1984, he was a Research Associate at Stanford University, where he worke on acoustic microscopy in superfluid helium. In 1984, he joined the IBM Research Division, San Jose, CA, where he is presently Manager of Nanoscale Studies. He has worked on many aspects of high-density data storage and scanning probe microscopy. His current research interests include ultrahigh density data storage, magnetic resonance force microscopy, and micromechanics. He has published more than 90 papers and holds 13 patents.

Dr. Rugar is currently a Distinguished Lecturer for the IEEE Magnetics Society and is a member of the American Physical Society. 